\title{
A Study on the Dependence of Water Tree Permittivity with Time
}

\author{
I. Radu $^{(1,2)}$, M. Acedo ${ }^{(1,3)}$, P. Notingher ${ }^{(1,2)}$, F. Frutos ${ }^{(3)}$ and J.C. Filippini ${ }^{(1)}$ \\ (1) Laboratoire d'Electrostatique et de Matériaux Diélectriques, C.N.R.S., B.P. 166, 38042 Grenoble, France \\ (2) Polytechnical University of Bucharest, 313 Splaiul Independentei, 77206 Bucharest, Romania \\ (3) Dpto. de Fisica Aplicada, Univ. de Sevilla, Avda. Reina Mercedes s/n. 41012 Sevilla, Spain
}

\begin{abstract}
During the growth of water trees in the insulation of a cable the distribution of the electric field is modified because of the local change of the dielectric properties of the material. It results a local enhancement of the electric field which could increase the risk of breakdown. The key factor is the permittivity of the water tree and the aim of the work is to determine its possible values and, particularly, the law of its increase with time during the growth of the trees. The paper presents permittivity measurements in uniform field laboratory models with water trees grown in accelerated conditions. The resulting variations of the electric field in the material are also given.
\end{abstract}

\section{Introduction}

Water treeing is a major cause of breakdown of the insulation of power cables. Several mechanisms have been proposed to explain how a water tree can lead to breakdown. One of these mechanisms consists in considering the dielectric nature of the water tree $[1,2$, 3]: since the average permittivity of a water tree is larger than that of the surrounding material, the ac electric field is lowered inside and enhanced outside the water tree. If a small defect exists in the vicinity of the front of the water tree the electric field at this defect may become high enough to initiate an electrical tree.

Such a mechanism should be governed by two factors: the geometry of the water tree and its permittivity $\varepsilon_{1}$. In order to examine its relevance it is important to know the value of the permittivity which plays a deciding role in the proposed mechanism.

Few studies have been devoted to the determination of $\varepsilon_{1}[1,4,5]$. It is known that $\varepsilon_{1}$ not only varies with the distance to the root of the tree but also that its value at each point is not constant with time. The objective of this work is precisely to determine the variations of $\varepsilon_{1}$ with time during the growth of the water tree.

\section{Water tree length measurements}

The study was performed using laboratory models made of crosslinked polyethylene and we chose the uniform field configurations for several reasons:

- a wide deteriorated zone can be generated if compared with the total insulation volume,

- very precise capacitance measurement can be obtained,

- the distribution of the water trees is similar to that found in MV and HV power cables.

The insulating material in which water trees were grown was in the form of plaques of XLPE of $0.5 \mathrm{~mm}$ thickness taken from a new HV power cable. The plaques were prealably sandblasted on one side in order to get a multitude of small inception points for water trees.

Then, they were bonded to polyethylene cylindrical reservoirs of inner diameter $2.5 \mathrm{~cm}$. The reservoirs were filled with a $0.1 \mathrm{~N}$ solution of $\mathrm{NaCl}$ in water. The voltage was applied through a platinum wire in contact with the liquid. The outer surface of the specimen was silver painted and grounded. The amplitude of the voltage was $5 \mathrm{kV}$ and the frequency was $10 \mathrm{kHz}$.

The growth kinetics of water trees was obtained using 18 identical specimens put under voltage for different periods of time from 10 hours to 180 hours.

After each period of time the active parts of three specimens were cut from the reservoir and rhodamine stained for 48 hours at $60^{\circ} \mathrm{C}$. Then, five $200 \mu \mathrm{m}$ width filament slices of each specimen were cut using a microtome in order to determine the number of trees, their lengths and their widths.

Sandblasting allows us to get a number of water trees growing from specific inception points and forming a quasi-continuous water tree layer. We characterized it by its maximum length, i.e. the length of the longer water tree and its average length calculated by 


$$
1=\frac{\sum_{i=1}^{n} l_{i} x_{i}}{x_{\max }}
$$

where $l_{i}$ and $x_{i}$ are respectively the length and width of every tree while $n$ stands for the number of trees of each slice and $x_{\max }$ the total length of the slice itself, as shown in figure 1.

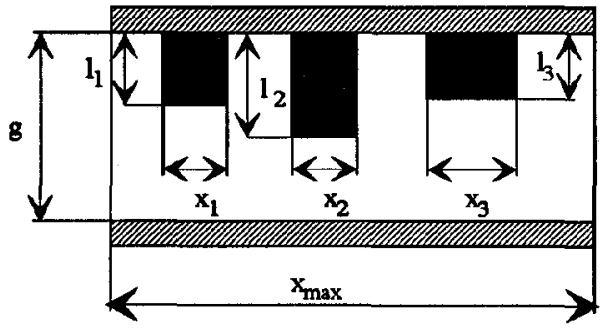

Figure 1. Model for average water tree length calculation.

Using equation (1) and making the arithmetic average value for the five filament slices taken out of 3 specimens for each period of time we determined the average length of the water trees as a function of time (figure 2).

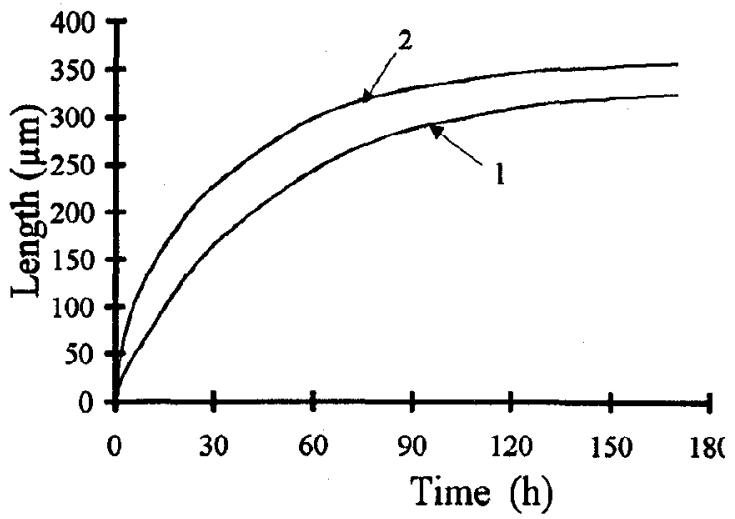

Figure 2. Time dependence of the average (1) and maximum (2) water tree length (water tree kinetics).

\section{Capacitance measurements}

The permittivity of the water trees was derived from the values of the capacitance of the specimens measured during the growth of the trees. The capacitance was measured on 5 identically aged specimens at different times between 0 and 180 hours of voltage application. The ageing conditions were the same as those used for the determination of the growth kinetics. A special test cell suited to the geometry of the specimens was developed at the CNRS/LEMD. It includes a shielding to minimize the electromagnetic effects and precise system with a guard ring to ensure a good reproducibility of the capacitance geometrical definition. The capacitance was measured using a General Radio bridge (model 1621) and an Irlab apparatus (model LDTRP-2). Figure 3 gives the time dependence of the capacitance $\mathrm{C}_{e}$ of the specimens during the growth of the water trees.

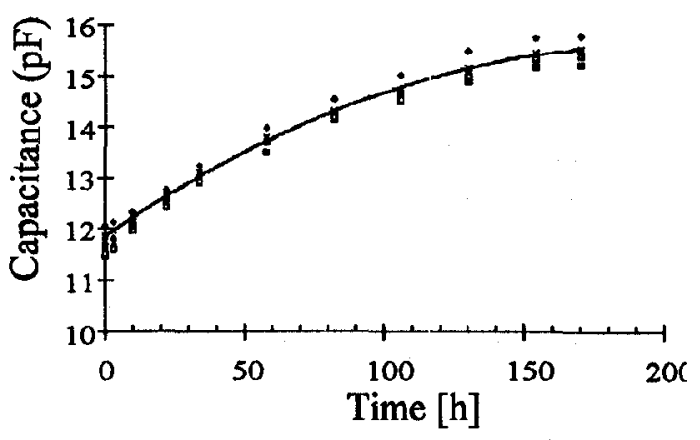

Figure 3. The variations of the capacitance C. of the specimens with time.

\section{Calculation of the permittivity}

Due to the high density of water trees of our sandblasted specimens we considered the water tree zone as a plane water tree layer of length $g_{1}=1$, average length of the water trees, and of average permittivity $\varepsilon_{1}$ and we modelled our specimen by two plane capacitors in series as shown in figure 4.

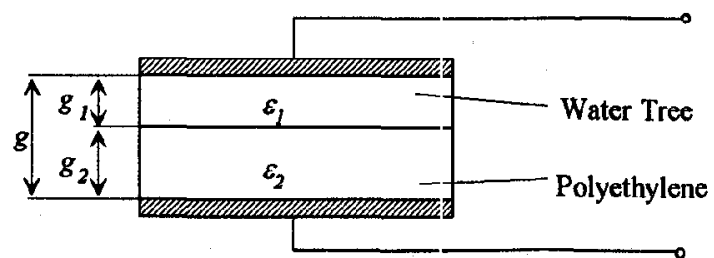

Figure 4. Equivalent plane capacitor model used for the calculation of the average permittivity fo the water trees.

The measured capacitance $\mathrm{C}_{\mathrm{e}}$ is connected to the capacitance $\mathrm{C}_{1}$ of the damaged area and to the capacitance $\mathrm{C}_{2}$ of the polyethylene zone without water tree by the relationship

$$
\frac{1}{\mathrm{C}_{e}}=\frac{1}{\mathrm{C}_{1}}+\frac{1}{\mathrm{C}_{2}}
$$


If $C_{i}=\varepsilon_{0} \varepsilon_{2} \mathrm{~S} / \mathrm{g}$ is the capacitance of the specimen before ageing, (2) gives the average permittivity $\varepsilon_{1}(t)$ of the treed zone:

$$
\varepsilon_{1}(t)=\frac{C_{e}(t) \varepsilon_{2} g_{1}(t)}{g_{1}(t) C_{e}(t)-g\left[C_{e}(t)-C_{i}\right]}
$$

Figure 5 shows the best fitted curve for average water tree permittivity as a function of time derived from (3), with $\varepsilon_{2}=2.3$ (polyethylene).

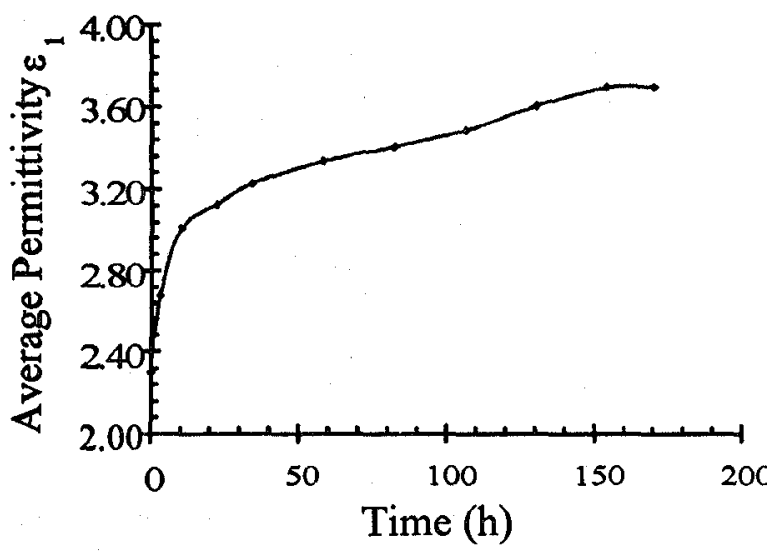

Figure 5. Average water tree permittivity as a function of ageing time.

Figure 6 shows the average permittivity as a function of the average water tree length.

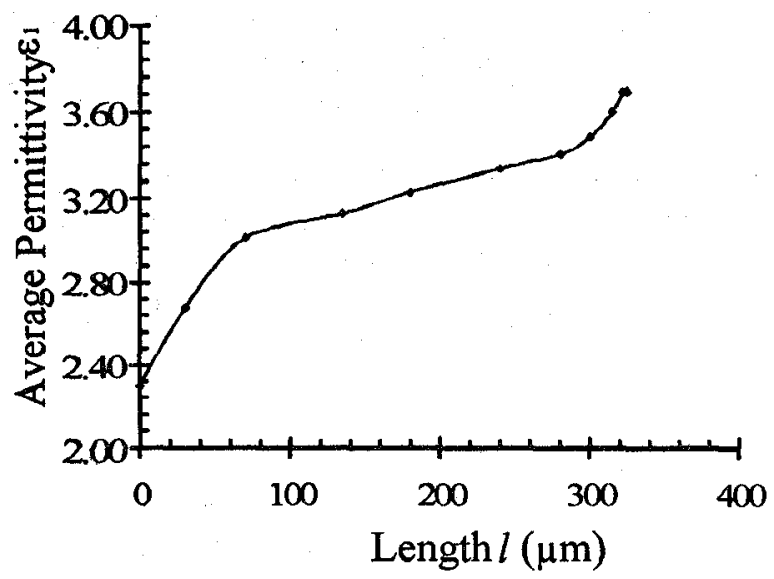

Figure 6. Average water tree permittivity as a function of water tree length.

From the time (or length) dependence of the average permittivity of the water trees we can derive the local value $\varepsilon_{\mathrm{r}}(\mathrm{y})$ of the permittivity as a function of the distance to the root of the water tree, if we know the variation law of $\varepsilon_{\mathrm{r}}$ inside the water tree.
We made the calculation assuming a linear dependence for the local permittivity:

$$
\begin{aligned}
& \varepsilon_{\mathrm{r}}(\mathrm{y})=\mathrm{ay}+\mathrm{b}, \mathrm{y} \in\left[0, \mathrm{~g}_{1}\right] \\
& \varepsilon_{\mathrm{r}}(\mathrm{y})=\mathrm{ag}_{1}+\mathrm{b}, \mathrm{y} \in\left[\mathrm{g}_{1}, \mathrm{~g}\right]
\end{aligned}
$$

where coefficients $a$ and $b$ are determined from our experimental and surrounding conditions:

$$
\varepsilon_{1}\left(g_{1}\right)=\frac{\int_{0}^{g_{1}} \varepsilon_{r}(y) d y}{g_{1}} ; \varepsilon_{2}=a g_{1}+b
$$

From equations (4) and (5) we obtained the local permittivity values for different times of voltage application. Figure 7 presents the results as a function of the distance $y$ to the water tree root for three stages of the water tree growth.

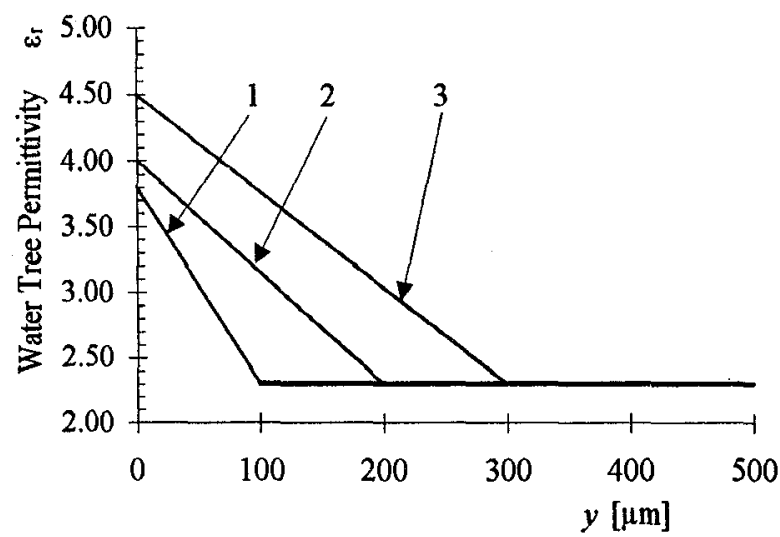

Figure 7. The variations of the local permittivity of the water tree zone as a function of the distance to the tree root for different times of voltage application: (1) $t=15 \mathrm{~h} ; \mathrm{l}=$ $100 \mu \mathrm{m}$; (2) $t=41 \mathrm{~h}, l=200 \mu \mathrm{m}$; (3) $t=106 \mathrm{~h}, \mathrm{l}=300 \mu \mathrm{m}$.

\section{Discussion}

As expected from observations of water trees during their growth [1] the average water tree permittivity is an increasing function of time. During the growth of the water tree, not only the length gets longer but also more water penetrates the material thus increasing the permittivity. The growth in permittivity is bigger in the early treeing stages, getting slower afterwards.

A plausible explanation of such a behaviour can be established if we look at the individual water trees on the successive cross-sections of our specimens. In early stages, as the degradation layer is not entirely uniform and a succession of individual trees can be still observed, water not only migrates through those trees 
but also forms new ones in their interstices. Both mechanisms lead to a quick average permittivity increase that slows down when the interstices are filled and water can only circulate through microchannels and microcavities of existing water trees.

In the last stages of the growth, the water tree length saturates but water goes on penetrating the material and the permittivity still increases as shown in figure 6.

Our average permittivity was found in the range 2.3 3.7 , the maximum value being obtained for an ageing period of $\mathbf{1 7 0}$ hours, with an average water tree length of $325 \mu \mathrm{m}$. Comparing with the few available data we notice that our maximum value lies between those of Stucki and Schönenberger [5] who found $\varepsilon=2.7$ to 3.6 and of those of Koo et al. [4] who found $\varepsilon=4$ to 6 . However, the experimental conditions were not the same in all experiments and comparison is not easy. In our experiments the high frequency of the voltage may have amplified the phenomenon. On the other hand, our assumption of an uniform tree layer leads to underevaluated permittivity values because of the untreed space inside the layer.

If we examine the consequences on the amplification of the electric field outside the treed layer, a calculation similar to those presented in [1] and [3] gives the curves presented in figure 8 . For this calculation we assumed a linear distribution of the permittivity. For the largest water tree layer $(1=300 \mu \mathrm{m})$ the electric field was found to be amplified by about $20 \%$.

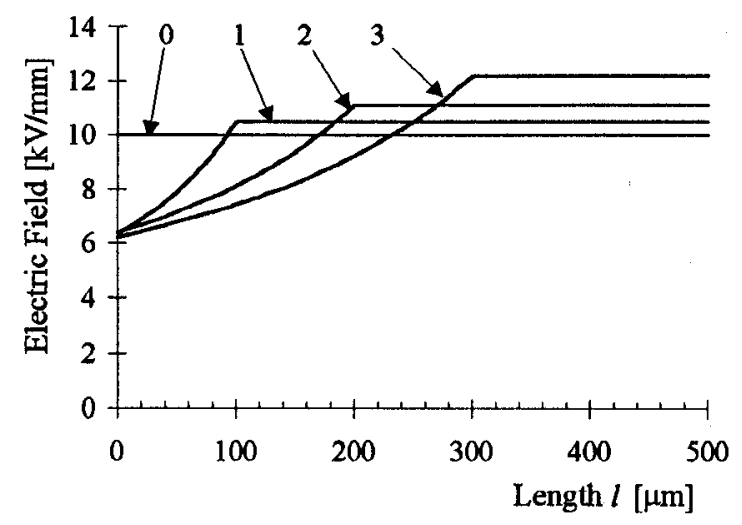

Figure 8. Electric field mapping in a 500 rm thick specimen subjected to a voltage of $5 \mathrm{kV}$ for different lengths of water tree regions: (0) without water tree; (l) $l=100 \mu \mathrm{m}$; (2) $l=200 \mu \mathrm{m}$; (3) $l=300 \mu \mathrm{m}$.

\section{Conclusion}

The time dependence of the average permittivity $\varepsilon_{1}$ of water trees in XIPE was experimentally determined during the growth of water trees in accelerated conditions.

$\varepsilon_{1}$ was found to change with time from 2.3 (nondamaged polyethylene) up to 3.7 (water tree of $325 \mu \mathrm{m}$ after 170 hours of ageing).

The increase in permittivity induces an enhancement of the electric field outside the water tree zone which could be a cause of breakdown. Even if the water tree length saturates, the permittivity continues to increase, thus increasing the risk of breakdown.

\section{Acknowledgment}

The authors are grateful to Mr G. Laulier of the CNRS/LEMD for excellent teclanical assistance.

\section{Referencies}

[1] J.L. Chen and J.C. Filippini "The morphology and behaviour of the water tree", IEEE Trans. E.I., pp 271-286, 1993.

[2] P. Notingher, I. Radu and J.C. Filippini, "Electric field calculations in polymers in the presence of water trees", $5^{\text {th }}$ Intern. Conf. on Conduction and Breakdown in Solid dielectrics, pp 666-670, 1995.

[3] M. Acedo, F. Frutos, M. Torres and J.C. Filippini, "The Cylindrical capacitive model for water treeing degradation in extruded H.V. cables", $5^{\text {th }}$ Intern. Conf. on Conduction and Breakdown in Solid Dielectrics, pp 661-665, 1995.

[4] J.Y. Koo, J.D. Cross, M. El Kahel, C.T. Meyer and J.C. Filippini, "Electrical Behaviour and Structure of Water trees in Relation to their propagation", Annual Reprort CEIDP, pp 301-306, 1983.

[5] F. Stucki and A. Schönenberger, "Dielectric properties of single water trees", $4^{\text {th }}$ Intern. Conf. on Conduction and Breakdown in Solid dielectrics, pp 373-377, 1992. 